\title{
X-ray dark-field and phase-contrast imaging using a grating interferometer
}

\author{
F. Pfeiffer, ${ }^{1, a)}$ M. Bech, ${ }^{2}$ O. Bunk, ${ }^{3}$ T. Donath, ${ }^{3}$ B. Henrich, ${ }^{3}$ P. Kraft, ${ }^{3}$ and C. David ${ }^{3}$ \\ ${ }^{1}$ Department of Physics, Technische Universität München, 85748 Garching, Germany \\ ${ }^{2}$ Niels Bohr Institute, University of Copenhagen, 2100 Copenhagen, Denmark \\ ${ }^{3}$ Paul Scherrer Institut, 5232 Villigen PSI, Switzerland
}

(Received 14 April 2008; accepted 8 August 2008; published online 19 May 2009)

\begin{abstract}
In this letter, we report results obtained with a recently developed approach for grating-based x-ray dark-field imaging [F. Pfeiffer et al., Nat. Mater. 7, 134 (2008)]. Since the image contrast is formed through the mechanism of small-angle scattering, it provides complementary and otherwise inaccessible structural information about the specimen at the micron and submicron length scales. Our approach is fully compatible with conventional transmission radiography and the grating-based hard X-ray phase-contrast imaging scheme [F. Pfeiffer et al., Nat. Phys. 2, 258 (2006)]. Since it can be used with standard $\mathrm{x}$-ray tube sources, we envisage widespread applications to X-ray medical imaging, industrial nondestructive testing, or security screening. (C) 2009 American Institute of Physics. [DOI: 10.1063/1.3115639]
\end{abstract}

\section{INTRODUCTION}

Visible light microscopy is a standard and widely utilized tool with a broad range of applications in science, industry, and everyday life. Besides standard bright-field imaging, many more contrast mechanisms have been developed, and dark-field imaging, phase-contrast, confocal, and fluorescence microscopies are routine methods in today's light microscopy applications. ${ }^{1}$ It is not surprising that this development has stimulated a similar progress in imaging applications with other forms of radiation. In electron microscopy, for example, where the initial electron microscope image was produced in the early 1930s, dark-field imaging was introduced in the late $1930 \mathrm{~s}^{2}{ }^{2}$ and imaging based on phase contrast in the $1940 \mathrm{~s}^{3}$

In x-ray microscopy or, more generally, x-ray imaging, the development of a similar range of contrast modalities proceeded much more slowly and is still a very active field of research. Despite the early pioneering work on x-ray interferometry by Bonse and Hart ${ }^{4}$ in the 1960s, the majority of phase-contrast imaging ${ }^{4-13}$ and dark-field imaging ${ }^{14-20}$ methods were introduced in the late 1990s. The development of such advanced imaging methods is particularly difficult for hard $\mathrm{x}$ rays (with energies in the the multi-keV range) because of the lack of efficient $\mathrm{x}$-ray optics. Existing hard $\mathrm{x}$-ray dark-field imaging methods, for example, rely on the use of crystal optics that can only accept a very narrow energy band width $(\approx 0.01 \%)$ and angular divergence ( $\approx 1$ arc sec) ${ }^{6,17-20}$ This is why dark-field or scattering-based imaging is currently restricted in practice to applications at highly brilliant synchrotron $\mathrm{x}$-ray sources ${ }^{16-20}$ and is not available for widespread applications that require a method applicable to standard $\mathrm{x}$-ray tubes. In the following we show results obtained with our recently developed grating-based dark-field imaging approach. ${ }^{21}$

\footnotetext{
${ }^{a)}$ Author to whom correspondence should be addressed. Electronic mail: franz.pfeiffer@ph.tum.de.
}

\section{PRINCIPLES}

Figure 1 shows our experimental arrangement. It consists of a source grating G0, a phase grating G1, and an analyzer absorption grating G2 [Fig. 1(a)]. The source grating (G0), typically placed close to the $\mathrm{x}$-ray tube, is an aperture mask with transmitting slits. It creates an array of periodically repeating line sources and effectively allows the use of relatively large, i.e., square-millimeter-sized x-ray sources, without compromising the coherence requirements

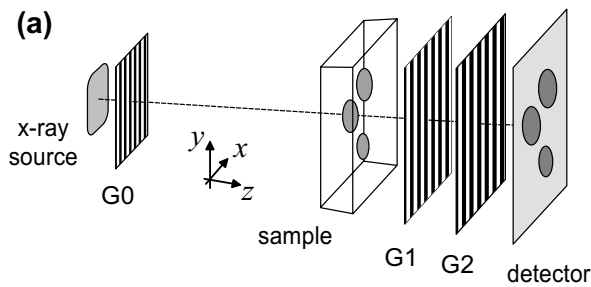

(b)

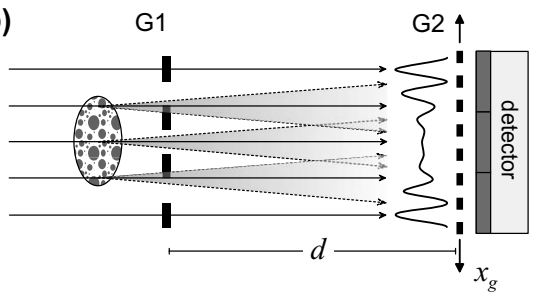

(c)

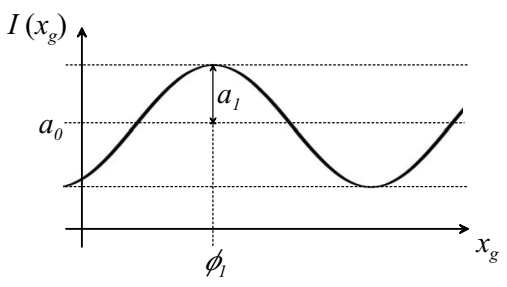

FIG. 1. X-ray grating interferometer. (a) Setup with a source grating G0, a phase grating G1, and an analyzer absorption grating G2. (b) Through the Talbot effect a linear periodic fringe pattern is created behind G1 in the plane of G2. (c) Intensity modulation detected in a detector pixel when one of the gratings is scanned along $x_{g}$. A loss in the amplitude of the oscillation due to the scattering of $\mathrm{x}$ rays in the specimen (degradation of the coherent wave front) can be used to extract images with dark-field contrast. 
of the arrangement formed by G1 and G2. The image contrast itself is formed via the combined effect of the two gratings G1 and G2. The second grating (G1) acts as phase mask and imprints periodic phase modulations onto the incoming wave field. Through the Talbot effect, ${ }^{22-25}$ the phase modulation is transformed into an intensity modulation in the plane of G2, forming a linear periodic fringe pattern perpendicular to the optical axis and parallel to the lines of G1 [Fig. 1(b)]. The third grating (G2) with absorbing lines and the same periodicity and orientation as the fringes created by G1 is placed in the detection plane, immediately in front of the detector. When one of the gratings is scanned along the transverse direction $x_{g}$, the intensity signal $I(m, n)$ in each pixel $(m, n)$ in the detector plane oscillates as a function of $x_{g}$ [Fig. 1(c)].

The fundamental idea of the method presented here is to evaluate the local changes in the oscillation $I\left(m, n, x_{g}\right)$ induced by an object and determine from these several imaging signals, including the dark-field contrast. To analyze these changes quantitatively, we write the intensity oscillation for each detector pixel in a Fourier series

$$
\begin{aligned}
I\left(m, n, x_{g}\right) & =\sum_{i} a_{i}(m, n) \cos \left[i k x_{g}+\phi_{i}(m, n)\right] \\
& \approx a_{0}(m, n)+a_{1}(m, n) \cos \left[k x_{g}+\phi_{1}(m, n)\right],
\end{aligned}
$$

where $a_{i}$ are the amplitude coefficients, $\phi_{i}$ are the corresponding phase coefficients, $k=2 \pi / p_{2}$, and $p_{2}$ is the period of G2. Then the normalized average transmission of the specimen in each detector pixel is given by $T(m, n)$ $=a_{0}^{s}(m, n) / a_{0}^{r}(m, n)$, where the superscripts $s$ and $r$ denote the values measured with the specimen in place $\left({ }^{s}\right)$ and as a reference without $\left({ }^{r}\right)$. Note that $T(m, n)$ is identical to what would be measured with a conventional $\mathrm{x}$-ray radiography setup. Furthermore, we have previously shown ${ }^{5}$ that differential phase-contrast images can be obtained by analyzing the lateral shift of intensity modulation, i.e., the quantity $\phi_{1}(m, n)$ in Eq. (1).

Dark-field images are based on the local scattering power of the sample and can also be obtained with such a setup. In terms of visible-light Fourier optics, dark-field illumination removes the zeroth order (unscattered light) from the diffraction pattern formed at the rear focal plane of the objective. This results in an image formed exclusively from higher angle diffraction intensities scattered by the specimen. Quite similarly, information about the scattering power of the specimen is contained in our case in the higher order, i.e., the first Fourier component of $I\left(m, n, x_{g}\right)$. More precisely, the amplitude of the first Fourier component, $a_{1}$, is decreased when $\mathrm{x}$ rays are scattered or reflected at internal inhomogeneities or interfaces on their passage through the specimen [Fig. 1(c)].

For a quantitative description of this effect, we first define the normalized oscillation amplitude, the visibility of the intensity modulation of $I\left(m, n, x_{g}\right)$, by the ratio

$$
V^{r}(m, n) \equiv \frac{I_{\max }^{r}-I_{\min }^{r}}{I_{\max }^{r}+I_{\min }^{r}}=\frac{a_{1}^{r}(m, n)}{a_{0}^{r}(m, n)},
$$

see Eq. (1). The relative decrease in the visibility due to the specimen can be quantified by defining the normalized visibility

$$
V(m, n) \equiv \frac{V^{s}(m, n)}{V^{r}(m, n)}=\frac{a_{0}^{r}(m, n) a_{1}^{s}(m, n)}{a_{0}^{s}(m, n) a_{1}^{r}(m, n)} .
$$

For homogeneous specimens, i.e., for samples with negligible small-angle $\mathrm{x}$-ray scattering (SAXS) contribution, the value for the visibility remains unchanged, and $V(m, n)=1$. But specimens, which, e.g., exhibit strong internal density fluctuations on micrometer length scales and thus produce a strong SAXS signal, show a significant decrease in the visibility and yield values of $V<1 .{ }^{24}$ More generally, we note that the quantity $V(m, n)$ is an inverse measure for the effective integrated local small (and ultrasmall)-angle scattering power of the sample and therefore refer to it as a "dark-field" image of the specimen. Most simply this can be understood by considering that the amplitude of the oscillations in $I\left(m, n, x_{g}\right)$ is most effectively reduced by $\mathrm{x}$ rays scattered from the object at wave vectors corresponding to diffraction angles defined by the ratio of half the period of G2 over the distance between the object and G2 [see also Fig. 1(b)].

\section{EXPERIMENTAL}

The experiments were carried out on a Seifert ID 3000 x-ray generator operated at $40 \mathrm{kV} / 25 \mathrm{~mA}$. We used a tungsten $(\mathrm{W})$ line focus tube $(\mathrm{DX}-\mathrm{W} 8 \times 0.4-\mathrm{L})$ with a focus size of 8 (horizontal) $\times 0.4$ (vertical) $\mathrm{mm}^{2}$. Due to the inclination of the target with respect to the optical axis of $6^{\circ}$, the effective source size was $0.8(h) \times 0.4(v) \mathrm{mm}^{2}$.

The gratings were fabricated by a process involving photolithography, deep etching into silicon, and electroplating of gold. ${ }^{26}$ The size of the active area was limited in the present study by the processing technology (100 $\mathrm{mm}$ wafers) to 64 $\times 64 \mathrm{~mm}^{2}$. The gratings had periods of $p_{0}=14 \mu \mathrm{m}, p_{1}$ $=3.5 \mu \mathrm{m}$, and $p_{2}=2.0 \mu \mathrm{m}$. The heights of the grating structures, which were optimized for a mean x-ray energy of 28 $\mathrm{keV}$, were $42 \mu \mathrm{m}(\mathrm{G} 0), 35 \mu \mathrm{m}(\mathrm{G} 1)$, and $26 \mu \mathrm{m}(\mathrm{G} 2)$. The distance between G0 and G1 was $1.4 \mathrm{~m}$ and between G1 and G2 $0.2 \mathrm{~m}$, corresponding to the fifth fractional Talbot distance. $^{25}$

The images were recorded using a PILATUS $100 \mathrm{~K}$ pixel detector. ${ }^{27}$ The module consists of an array of 487 $\times 195$ pixels with a pixel size of $0.172 \times 0.172 \mathrm{~mm}^{2}$. For the results displayed in Fig. 3, several images were stitched together to increase the effective field of view. The quantum detection efficiency is determined by the probability of absorbing an $\mathrm{x}$ ray in the $320 \mu \mathrm{m}$ thick Si sensor and is $\simeq 10 \%$ (at $28 \mathrm{keV}$ ). For the results shown in Figs. 2 and 3, a series of eight individual images with exposure times of $10 \mathrm{~s}$ each was recorded.

To extract the dark-field, phase-contrast, and absorptioncontrast signals from the measured intensity modulation $I\left(m, n, x_{g}\right)$, one-dimensional discrete fast Fourier transforms were computed for each pixel. The resulting values for the 

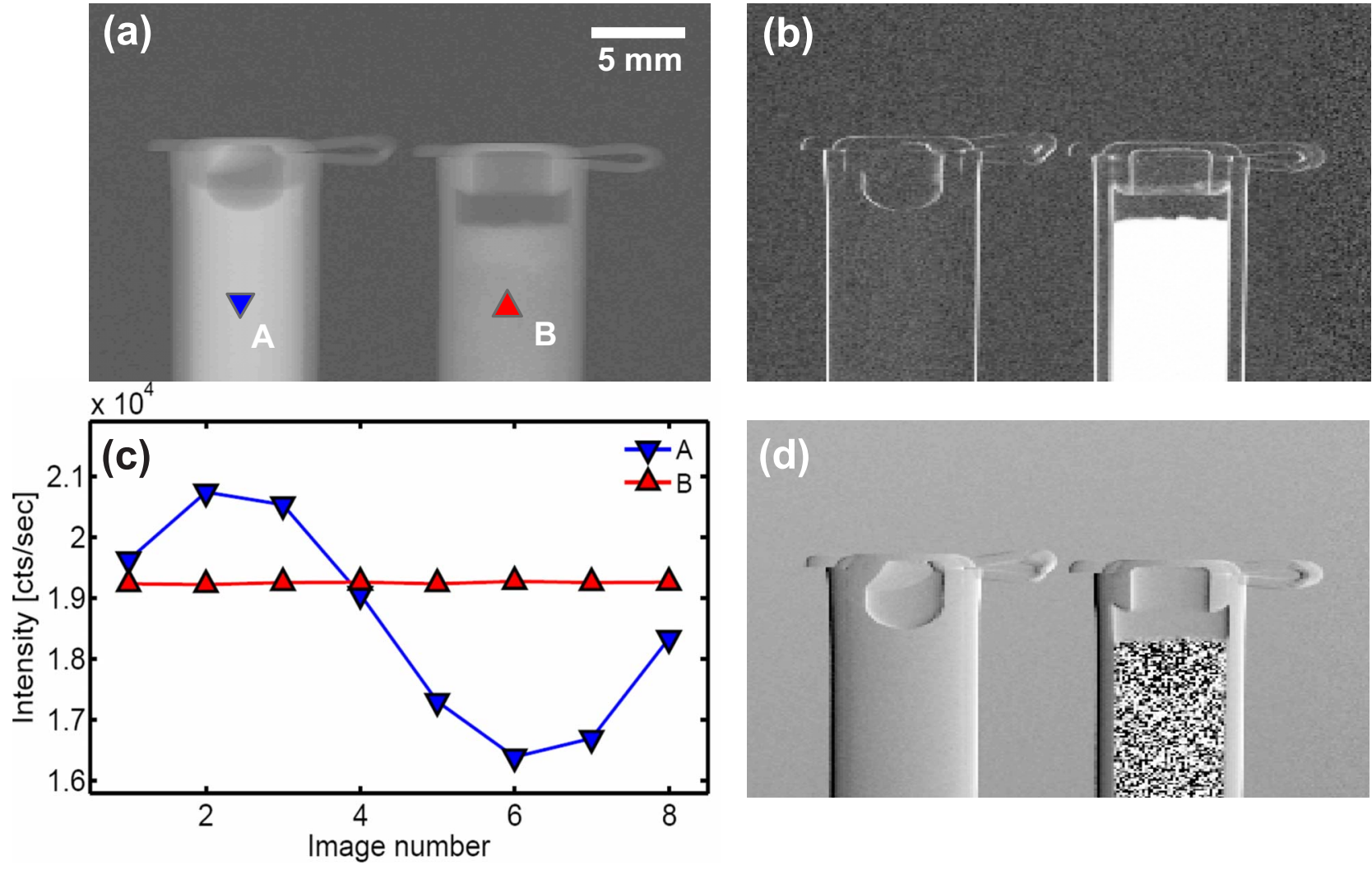

FIG. 2. (Color online) X-ray images of a test sample containing two plastic containers filled with a liquid (water, left, A) and a powder (sugar, right, B). (a) Conventional x-ray transmission image. (b) Dark-field image of the same sample. (c) Intensity oscillations observed in detector pixels corresponding to two regions of the sample, extracted from a series of eight images taken at different values of $x_{g}$ (averaged over 50 pixels). (d) Differential phase-contrast image.

Fourier coefficients were normalized to the corresponding values obtained without specimen in place. On a standard personal computer (1.7 GHz processor, 2 Gbyte memory), the processing time needed for a series of eight images with $10^{5}$ pixels was on the order of a few seconds.

\section{RESULTS}

Figure 2 displays the experimental results obtained for a test sample comprising two plastic containers filled with a liquid (water) and a powder (sugar). The conventional transmission image is shown in Fig. 2(a), the dark-field image in Fig. 2(b), and the differential phase-contrast image in Fig. 2(d). The two substances (water and sugar) both absorb some fraction of the incident $\mathrm{x}$-ray beam and therefore show up in the transmission image [Fig. 2(a)]. In contrast, a quite different picture is observed in the dark-field image [Fig. 2(b)], where only the powder sample (and the edges of the plastic containers) produced a strong contrast. This is because the microscopic density fluctuations present in the powder produce a strong small-angle scattering signal. Thus the intensity modulations in $I\left(m, n, x_{g}\right)$ [Fig. 2(c)] are lowered significantly, and a strong contrast is produced in the the dark-field image [Fig. 2(b)]. The liquid, on the other hand, shows no significant contributions to the dark-field signal since water is a homogeneous material with essentially no density fluctuations on the relevant length scales. The fact that the powder very effectively lowers the visibility in the corresponding pixels is also reflected in the differential phase-contrast image shown in Fig. 2(d). In the region, where the visibility is almost zero, the algorithm can no longer determine the phase-contrast signal correctly and yields a random distribution of black and white pixels [behind the strongly scattering powder, see Fig. 2(d)]. The small-angle scattering signal as recorded in the dark-field image is particularly sensitive to density variations in the object on the length scale of a few tenths of nanometers to several micrometers. It naturally complements the length scales which can be imaged directly by radiographic methods into the submicrometer range.

Figure 3 displays results of imaging a biological specimen, i.e., the wing of a blackbird (postmortem). The conventional absorption-contrast image is shown in Fig. 3(a), the dark-field image in Fig. 3(b), and the phase-contrast image in Fig. 3(c). Note that, since all three images were obtained from the same data set, the radiation dose was identical in all three cases. In agreement with what was observed in the images of the test sample shown in Fig. 2, we find that also in this biological specimen the boundaries and interfaces produce a strong signal in the dark-field image [see Fig. 3(b)]. This is particularly reflected in the high contrast produced by the detailed fine structure of, for example, the feathers in the wing. They obviously consist of a light, but highly structured and strongly scattering fiber network since they are clearly visible in the dark-field contrast [Fig. 3(b)]. These details are difficult to see in the absorption image [Fig. 3(a)]. The bones in the wing, however, are very nicely resolved in the absorption image [Fig. 3(a)]. The phasecontrast image [Fig. 3(c)] complements the details visible in the dark-field and absorption image by highlighting, in par- 

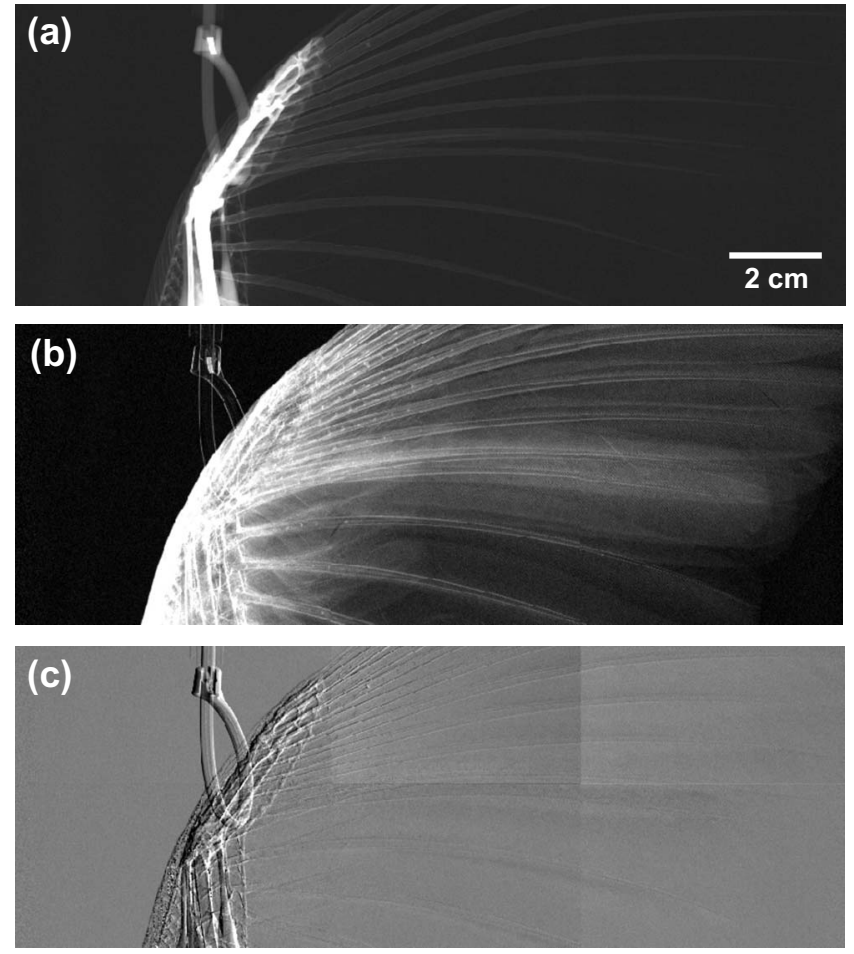

FIG. 3. Postmortem images obtained for a biological specimen (wing of a blackbird). (a) Conventional transmission image. (b) Dark-field image. (c) Differential phase-contrast image.

ticular, the edges of the core structure of the wing.

\section{SUMMARY AND OUTLOOK}

In summary, we have shown how a grating interferometer can be used to simultaneously produce dark-field, phasecontrast, and the conventional absorption images with hard $\mathrm{x}$ rays. We have demonstrated that, in contrast to existing crystal analyzer based methods, ${ }^{17-20}$ our method can efficiently be implemented with a standard $\mathrm{x}$-ray tube source. The approach is potentially interesting for a wide range of applications including medical imaging, ${ }^{20}$ security screening, ${ }^{28}$ industrial nondestructive testing, ${ }^{29}$ food inspection, and small animal imaging. In the future, a further increase in the sensitivity of the method could also provide contrast between healthy and diseased breast tissue since breast tumors exhibit a different small-angle scattering signature than the healthy adipose matrix. ${ }^{30,31}$ Generally, we believe that the method is of particular interest for challenging $\mathrm{x}$-ray imaging applications because it simultaneously provides dark field, transmission, and differential phase contrast, thus providing maximum information about the specimen. Finally, this approach can be extended into three dimensions using computer tomographic techniques, ${ }^{32}$ coupled with a synchrotron source to yield micrometer-resolution hard $\mathrm{x}$-ray dark-field images ${ }^{33}$ or implemented with other types of radiation, such as neutrons. ${ }^{34}$

\section{ACKNOWLEDGMENTS}

We gratefully acknowledge C. Grünzweig for the grating fabrication, J. Herzen for assistance during the experiments, and Ch. Brönnimann and E. F. Eikenberry for providing the detector. F. P. acknowledges support through the DFG Cluster of Excellence Munich-Center for Advanced Photonics.

${ }^{1}$ D. B. Murphy, Fundamentals of Light Microscopy and Electronic Imaging (Wiley, New York, 2001).

${ }^{2}$ M. von Ardenne, Z. Tech. Phys. (Leipzig) 19, 407 (1938).

${ }^{3}$ E. G. Ramberg, J. Appl. Phys. 20, 441 (1949).

${ }^{4}$ U. Bonse and M. Hart, Appl. Phys. Lett. 6, 155 (1965).

${ }^{5}$ F. Pfeiffer, T. Weitkamp, O. Bunk, and C. David, Nat. Phys. 2, 258 (2006).

${ }^{6}$ T. J. Davis, D. Gao, T. E. Gureyev, A. W. Stevenson, and S. W. Wilkins, Nature (London) 373, 595 (1995).

${ }^{7}$ V. N. Ingal and E. A. Beliaevskaya, J. Phys. D: Appl. Phys. 28, 2314 (1995).

${ }^{8}$ A. Snigirev, I. Snigireva, V. Kohn, S. Kuznetsov, and I. Schelokov, Rev. Sci. Instrum. 66, 5486 (1995).

${ }^{9}$ S. W. Wilkins, T. E. Gureyev, D. Gao, A. Pogany, and A. W. Stevenson, Nature (London) 384, 335 (1996).

${ }^{10}$ D. Chapman, Phys. Med. Biol. 42, 2015 (1997).

${ }^{11}$ A. Momose, Jpn. J. Appl. Phys., Part 1 44, 6355 (2005).

${ }^{12}$ A. Momose, W. Yashiro, Y. Takeda, Y. Suzuki, and T. Hattori, Jpn. J. Appl. Phys., Part 1 45, 5254 (2006).

${ }^{13}$ T. Weitkamp, A. Diaz, C. David, F. Pfeiffer, M. Stampanoni, P. Cloetens, and E. Ziegler, Opt. Express 13, 6296 (2005).

${ }^{14}$ G. R. Morrison and M. T. Browne, Rev. Sci. Instrum. 63, 611 (1992).

${ }^{15}$ H. N. Chapman, C. Jacobsen, and S. Williams, Ultramicroscopy 62, 191 (1996).

${ }^{16}$ Y. Suzuki and F. Uchida, Rev. Sci. Instrum. 66, 1468 (1995).

${ }^{17}$ E. Pagot, S. Fiedler, P. Cloetens, A. Bravin, P. Coan, K. Fezzaa, J. Baruchel, and J. Härtwig, Appl. Phys. Lett. 82, 3421 (2003).

${ }^{18}$ L. E. Levine and G. G. Long, J. Appl. Crystallogr. 37, 757 (2004).

${ }^{19}$ M. Ando, E. Hashimoto, H. Hashizume, K. Hyodo, H. Inoue, T. Kunisada, A. Maksimenko, K. Mori, E. Rubenstein, J. Roberson, D. Shimaoc, H. Sugiyama, K. Takeda, F. Toyofuku, E. Ueno, K. Umetani, H. Wada, and W. Pattanasiriwisawa, Nucl. Instrum. Methods Phys. Res. A 548, 1 (2005).

${ }^{20}$ D. Shimao, H. Sugiyama, T. Kunisada, and M. Ando, Appl. Radiat. Isot. 64, 868 (2006).

${ }^{21}$ F. Pfeiffer, M. Bech, O. Bunk, P. Kraft, E. F. Eikenberry, C. Brönnimann, C. Grünzweig, and C. David, Nature Mater. 7, 134 (2008).

${ }^{22}$ A. W. Lohmann and D. E. Silva, Opt. Commun. 2, 413 (1971).

${ }^{23}$ S. Yokozeki and T. Suzuki, Appl. Opt. 10, 1575 (1971).

${ }^{24}$ F. Pfeiffer, O. Bunk, C. Schulze-Briese, A. Diaz, T. Weitkamp, C. David, J. F. van der Veen, I. Vartanyants, and I. K. Robinson, Phys. Rev. Lett. 94, 164801 (2005).

${ }^{25}$ T. Weitkamp, C. David, C. Kottler, O. Bunk, and F. Pfeiffer, SPIE Int., Soc. Opt. Eng. 6318, 28 (2006).

${ }^{26}$ C. David, J. Bruder, T. Rohbeck, C. Grunzweig, C. Kottler, A. Diaz, O. Bunk, and F. Pfeiffer, Microelectron. Eng. 84, 1172 (2007).

${ }^{27}$ M. Bech, O. Bunk, C. David, P. Kraft, C. Brönnimann, E. F. Eikenberry, and F. Pfeiffer, Appl. Radiat. Isot. 66, 474 (2008).

${ }^{28}$ G. Harding, Radiat. Phys. Chem. 71, 869 (2004).

${ }^{29}$ G. Harding and B. Schreiber, Radiat. Phys. Chem. 56, 229 (1999).

${ }^{30}$ M. Fernandez, J. Keyrilainen, R Serimaa, M. Torkkeli, M.-L. KarjalainenLindsberg, M. Tenhunen, W. Thomlinson, V. Urban, and P. Suortti, Phys. Med. Biol. 47, 577 (2002).

${ }^{31}$ M. Fernández, J. Keyriläinen, R. Serimaa, M. Torkkeli, M.-L. KarjalainenLindsberg, M. Leidenius, K. von Smitten, M. Tenhunen, S. Fiedler, A. Bravin, T. M. Weiss, and P. Suortti, Phys. Med. Biol. 50, 2991 (2005).

${ }^{32}$ F. Pfeiffer, C. Kottler, O. Bunk, and C. David, Phys. Rev. Lett. 98, 108105 (2007).

${ }^{33}$ F. Pfeiffer, O. Bunk, C. David, M. Bech, G. Le Duc, A. Bravin, and P. Cloetens, Phys. Med. Biol. 52, 6923 (2007).

${ }^{34}$ F. Pfeiffer, C. Grunzweig, O. Bunk, G. Frei, E. Lehmann, and C. David, Phys. Rev. Lett. 96, 215505 (2006). 